\title{
Vinazas de mezcal y su efecto biofertilizante en el rendimiento de hortalizas
}

\section{Mezcal vinases and their fertilizing effect on the yield of vegetables}

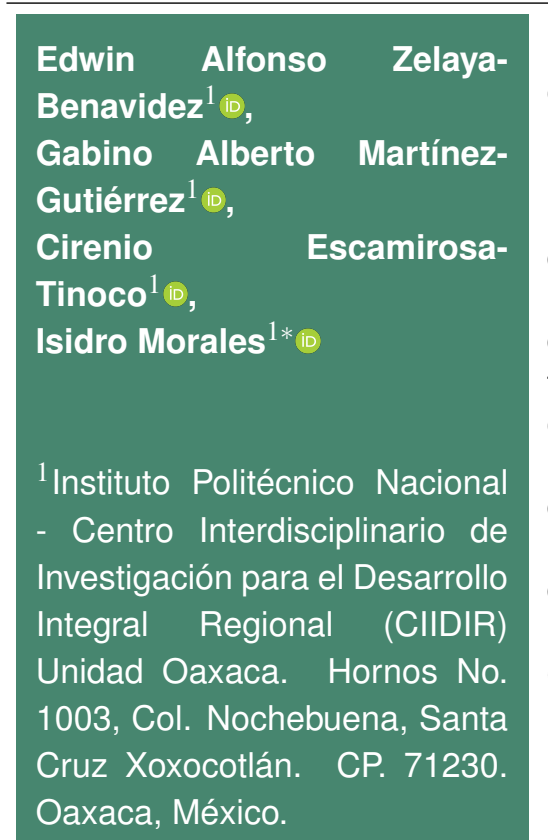

*Autor de correspondencia: imoralesg@ipn.mx

Artículo científico

Recibido: 20 de octubre 2021

Aceptado: 09 de diciembre 2021

Como citar: Zelaya-Benavidez EA, Martínez-Gutiérrez GA, Escamirosa-Tinoco C, Morales I (2021) Vinazas de mezcal y su efecto biofertilizante en el rendimiento de hortalizas. Ecosistemas y Recursos Agropecuarios 8(3): e3159. DOI: 10.19136/era.a8n3.3159
RESUMEN. Las vinazas de mezcal son residuos líquidos recalcitrantes con alta carga orgánica y elementos disueltos, la cual puede ser utilizada en la nutrición de plantas. El objetivo del trabajo fue evaluar la toxicidad de las vinazas del mezcal en la germinación de semillas y su efecto biofertilizante en el rendimiento de hortalizas. Se realizaron dos experimentos en lechuga, tomate y pepino: 1) prueba de fitotoxicidad en la germinación en semillas con diferentes concentraciones de vinaza $(2.5,5$, $10,20,40,80$ y $100 \%)$; y 2). Se evaluó el efecto biofertilizante de las combinaciones de $100-0,80-20,40-60$ y $20-80 \%$ de vinaza y solución nutritiva Steiner, y como testigo el $100 \%$ de la solución nutritiva; en ambos experimentos se utilizó un diseño completamente al azar. La concentración del $2.5 \%$ de vinaza del mezcal, fue tóxica para las semillas de lechuga y más del $10 \%$ inhibió su germinación. Para semillas de tomate el $20 \%$ resultó tóxico, mientras que en semillas de pepino provocó hormesis. La concentración del $80 \%$ de vinazas inhibió la germinación en semillas de tomate. La combinación: $40+60 \%$ de vinaza y solución nutritiva aplicados en la nutrición de lechuga y tomate, alcanzó los máximos valores de todas las variables evaluadas. En pepino, el rendimiento disminuyó de forma lineal al aumentar el porcentaje de vinaza como complemento de la solución nutritiva.

Palabras clave: Biofertilización, fitotoxicidad, residuos de agave, sustrato, solución nutritiva.

ABSTRACT. Mezcal vinasses are recalcitrant liquid waste with a high organic load and dissolved elements that can be used in plant nutrition. The objective work was to evaluate the toxicity of mezcal vinasse on seed germination and its biofertilizing effect on vegetable yield. Two experiments were carried out on lettuce, tomato and cucumber: 1) germination phytotoxicity test in seeds with different concentrations of vinasse $(2.5,5,10,20,40,80$ and 100\%); and 2) The biofertilizing effect of the combinations of $100-0,80-20,40-60$ and $20-80 \%$ vinasse and Steiner nutrient solution was evaluated, and $100 \%$ of the nutrient solution as a control. A completely randomized design was used in both experiments. The $2.5 \%$ concentration of mezcal vinasse was toxic to lettuce seeds and greater than $10 \%$ inhibited their germination. For tomato seeds the $20 \%$ was toxic, while in cucumber seeds it caused hormesis. The $80 \%$ concentration of vinasses inhibited germination in tomato seeds. The combination: $40+60 \%$ vinasse and nutrient solution applied in the nutrition of lettuce and tomato, reached the maximum values of all the variables evaluated. In cucumber, the yield decreased linearly with increasing the vinasse percentage as a supplement to the nutrient solution.

Key words: Agave residues, biofertilization, phytotoxicity, nutrient solution, substrate. 


\section{INTRODUCCIÓN}

En los nueve estados de la República Mexicana con denominación de origen del mezcal, se producen anualmente cinco millones de litros de mezcal, de los cuales 4.5 millones ( $90 \%$ ), son producidos en Oaxaca (CRM 2019). Cada litro de mezcal producido artesanal o industrialmente genera, como subproducto de la fermentación y destilación del agave (Rodríguez y De La Cerna 2017), entre 10 y 12 litros de vinazas (Beltrán et al. 2001). Lo que indica que, se vierten al medio ambiente anualmente y sin tratamiento alguno, entre 45 y 54 millones de litros de vinazas, las cuales son residuos líquidos recalcitrantes, con pH ácido y alta carga orgánica (RoblesGonzález et al. 2018).

El vertido al suelo de este residuo $\sin$ tratamiento, como sale del palenque, puede tener efectos tóxicos para el crecimiento de plantas sensibles a la salinidad y nocivo para los microorganismos del suelo como las micorrizas (Sánchez-Lizárraga et al. 2018). Al respecto, Sánchez-Lizárraga et al. (2018) evaluaron la aplicación de vinazas del tequila al suelo con efectos adversos en la población de micorrizas, aumento de la salinidad y disminución del crecimiento de plantas araña (Chlorophytum comosum, (Thunb.) Jacques), encontrando que el efecto fitotóxico de las vinazas se debe al incremento de la concentración de sales en el suelo.

Las vinazas presentan propiedades que pueden resultar nocivas como $\mathrm{pH}$ ácido, alta concentración de sales, fenoles, compuestos aromáticos (Robles-González et al. 2018), alcoholes, ácidos orgánicos (acético, isobutírico, butírico, valérico, capróico, enántico, caprílico, láurico, palmítico y otros), furanos, alcanos, aldehídos, ésteres, cetonas, y piranos (Rodríguez-Félix et al. 2018). Pero también se ha demostrado que las vinazas del mezcal y del tequila contienen elementos como calcio, fósforo, magnesio, nitrógeno y azufre (López-Velarde et al. 2020), además de materia orgánica (RoblesGonzález et al. 2012). Por lo que, se aplican en el suelo (Morán-Salazar et al. 2016) o para el mejoramiento nutrimental de compostas (López-López et al. 2010). De igual manera, el uso de vinazas del etanol de caña de azúcar diluida al 10\% como complemento de una solución nutritiva en el cultivo hidropónico de lechuga, mostró resultados positivos en su crecimiento (Dos Santos et al. 2013).

Para reutilizar las vinazas como complemento en la nutrición de cultivos de interés económico, es necesario evaluar su posible fitotoxicidad en diferentes concentraciones. Los bioensayos de germinación en semillas ofrecen indicadores de la respuesta ante sustancias tóxicas contenidas en los residuos (Mendes et al. 2021), lo cual puede inhibir o estimular puntos de crecimiento vegetal y elongación o disminución de la raíz (López-Diazguerrero et al. 2013). Al respecto, Da Ros et al. (2018), con el fin de determinar el potencial del digestato procedente de precipitados del vino para su uso como fertilizante agrícola, evaluaron su fitotoxicidad en macrófitas dicotiledóneas (Lepidium sativum L. y Sinapis alba L.) y una monocotiledónea (Sorghum saccharatum L.). También, Viveros-Legorreta et al. (2018) determinaron la fitotoxicidad de fenoles en lechuga ( $L a c-$ tuca sativa L.) liberados por Myriophyllum aquaticum (Vell.) Verdc. en agua, con efecto estimulante $u$ hormesis en la elongación radical de esta especie. También Castro-Rivera et al. (2020) determinaron el efecto fitotóxico en lechuga del digestato de un biodigestor alimentado con estiércol de bovino y residuos de la cosecha de tomate. Mientras que RodríguezRomero et al. (2014) al evaluar la calidad del agua de un río contaminado con aguas residuales municipales mediante un ensayo de fitotoxicidad en lechuga encontraron que la elongación radicular fue mayor ante altas concentraciones de nutrientes, pero la germinación fue inhibida por la presencia de nitrógeno amoniacal. En tanto que Sousa et al. (2019) al evaluar la fitotoxicidad de vinazas del vino en ajo (Allium cepa L.), lechuga (L. sativa L.), berro (Lepidium sativum L.), tomate (Solanum lycopersicum L.), frijol (Phaseolus vulgaris L.) y maíz (Z. mays L.) encontraron que concentraciones de vinazas superiores al $20 \%$ fueron tóxicas. Mientras que CastroLizazo et al. (2021) estimaron el efecto fitotóxico y fertilizante de vinazas del vino en el cultivo de tomate. Pero el efecto fitotóxico y biofertilizante de las vinazas del mezcal, en cultivos hortícolas de im- 
portancia económica como el tomate ( $S$. lycopersicum L.) (Martínez-Damián et al. 2019), sensible y tolerante a la salinidad como lechuga (L. sativa L.) y pepino (Cucumis sativus L.), aún no se evalúa. Con base en lo anterior, el objetivo del estudio fue evaluar la toxicidad de las vinazas del mezcal en la germinación de semillas y su efecto biofertilizante en el rendimiento de lechuga, tomate y pepino, en cultivo sin suelo e invernadero.

\section{MATERIALES Y MÉTODOS}

Se realizaron dos experimentos independientes. El primero consistió en una prueba de germinación en semillas de lechuga ( $L$. sativa L.) cv. Roma $^{\circledR}$, tomate (S. lycopersicum L.) cv. Pony express F1 (Harris Moran ${ }^{\circledR}$ ) de habito de crecimiento determinado y pepino (C. sativus L.) cv. Americano ${ }^{\circledR}$, utilizando diferentes diluciones de vinaza de mezcal y agua. En el segundo, se evaluó la vinaza como complemento de la solución nutritiva en el rendimiento de tomate, pepino y lechuga. Los dos experimentos se hicieron en el Centro Interdisciplinario de Investigación para el Desarrollo Integral Regional, del Instituto Politécnico Nacional (CIIDIR IPN, Unidad Oaxaca), en Santa Cruz Xoxocotlán, Oaxaca, México ( $17^{\circ} 01^{\prime} 30.3^{\prime \prime}$ LN y $96^{\circ} 43^{\prime} 12.5^{\prime \prime}$ LO, a $1530 \mathrm{msnm})$.

\section{Obtención de muestras}

La vinaza se obtuvo de la fábrica de mezcal San Pablo, en el municipio de San Pablo Villa de Mitla, Oaxaca, México ( $16^{\circ} 55^{\prime} \mathrm{LN}$ y $96^{\circ} 22^{\prime} \mathrm{LO}$, a 1 $680 \mathrm{msnm}$ ), como sale del palenque y depositada en un contenedor de $600 \mathrm{~L}$ para su posterior uso.

\section{Experimento 1}

De la vinaza colectada, se tomaron $2 \mathrm{~L}$, que se homogenizaron y filtraron en papel filtro Whatman grado 1 , de este filtrado se tomó una muestra de $800 \mathrm{~mL}$ para su análisis. Se determinó el pH y la conductividad eléctrica (CE) con un potenciómetro y conductímetro integrado digital marca Hanna (Hanna Instruments ${ }^{\circledR}$ ) mediante la metodología propuesta por Visconti y De Paz (2018). Los resultados fueron:
pH 3.84 y CE $3.22 \mathrm{dS} \mathrm{m}^{-1}$. El contenido de nutrientes de la vinaza se obtuvo por los métodos de la AOAC (AOAC 2003), en meq $\mathrm{L}^{-1}$ con valores de: $\mathrm{NO}_{3^{-}} 0.68, \mathrm{H}_{2} \mathrm{PO}_{4} 2.20, \mathrm{~K}^{+} 1.10, \mathrm{Ca}^{2+} 43.30 \mathrm{y} \mathrm{Mg}^{2+}$ 20.80 .

El efecto fitotóxico de la vinaza se determinó en función de los índices de germinación normalizado (IGN) e índice de elongación radical (IER), así como con los valores de fitotoxicidad, propuestos por Bagur-González et al. (2011). Los IGN e IER se obtuvieron con las siguientes ecuaciones:

$$
I G N=\frac{\text { Germx }- \text { GermTestigo }}{\text { GermTestigo }}
$$

Donde: Germx: es el porcentaje promedio de semillas germinadas en cada dilución de vinaza y GermTestigo: es el porcentaje de semillas germinadas en el testigo.

$$
I E R=\frac{\text { Elong } x-\text { ElongTestigo }}{\text { ElongTestigo }}
$$

Donde: Elongx: es la longitud promedio de la radícula de las semillas germinadas en dilución de vinaza y ElongTestigo: es la longitud promedio de la radícula de las semillas germinadas en el testigo.

El rango de fitotoxicidad fue de acuerdo con Bagur-González et al. (2011) y consistió en: mayor que 0 , hormesis o estimula el crecimiento, de 0 a 0.25 baja toxicidad, de -0.25 a -0.50 moderadamente tóxico, de -0.50 a -0.75 muy tóxico de -0.75 a -1.00 altamente tóxico. Para esta prueba, las semillas de las tres especies se depositaron en placas Petri sobre papel filtro grado 1 (Whatman) humedecido con 7 $\mathrm{mL}$ de cada dilución. Se hicieron seis diluciones de vinaza en agua que correspondieron a los tratamientos (Tabla 1) y como testigo absoluto; únicamente agua destilada y testigo relativo, la vinaza al $100 \%$ sin diluir, con cuatro repeticiones por tratamiento y 15 semillas por repetición de acuerdo con Wang (1991).

\section{Experimento 2}

Se utilizaron semillas de L. sativa L., $S$. lycopersicum L. y $C$. sativus $L$., de las variedades utilizadas en el experimento 1, las cuales germinaron en charolas de poliestireno expandido de 200 cavidades, 
utilizando como sustrato una mezcla de turba Sphagnum + Agrolita (70:30 v/v); treinta días después de la germinación, se realizó el trasplante a bolsas de polietileno negro de $18 \mathrm{~L}$ que tenían como sustrato bagazo de agave mezcalero compostado y cribado, cuyo tamaño de partícula fue de $0.42-1.18 \mathrm{~mm}$, CE $1.37 \mathrm{dS} \mathrm{m}^{-1}$ y pH 5.32, de acuerdo a lo recomendado por Martínez-Gutiérrez et al. (2013). Las bolsas se colocaron en un invernadero tipo multicapilla de $10 \times 20 \times 4.50 \mathrm{~m}$ de ancho largo y alto, respectivamente. La densidad de siembra fue de 5, 4 y 2.5 plantas $\mathrm{m}^{-2}$, para lechuga, tomate y pepino, en el mismo orden. Por presentar diferentes métodos de manejo de cultivo, cada hortaliza se agrupó de manera independiente y las tres especies estudiadas estuvieron contiguas. En cada grupo, la distribución fue completamente al azar con cinco repeticiones por tratamiento, cada unidad experimental consistió en dos plantas por bolsa para tomate y lechuga, y una para pepino.

Tabla 1. Potencial de hidrógeno $(\mathrm{pH})$ y conductividad eléctrica de las diluciones de vinaza del mezcal utilizadas en la prueba de germinación de semillas de lechuga, tomate y pepino.

\begin{tabular}{ccc}
\hline$\%$ de vinaza & $\mathrm{pH}$ & Conductividad eléctrica $\left(\mathrm{dS} \mathrm{m}^{-1}\right)$ \\
\hline 0 & $5.46 \pm 0.11$ & $0.01 \pm 0.00$ \\
2.5 & $3.88 \pm 0.01$ & $0.53 \pm 0.01$ \\
5 & $3.83 \pm 0.01$ & $0.97 \pm 0.07$ \\
10 & $3.82 \pm 0.01$ & $1.49 \pm 0.03$ \\
20 & $3.78 \pm 0.01$ & $2.51 \pm 0.06$ \\
40 & $3.75 \pm 0.01$ & $5.59 \pm 0.13$ \\
80 & $3.72 \pm 0.02$ & $9.14 \pm 0.10$ \\
100 & $3.67 \pm 0.01$ & $10.91 \pm 0.26$ \\
\hline$:$ desviación estándar.
\end{tabular}

El contenido de elementos de la vinaza y del agua de riego se determinó por los métodos de la AOAC (AOAC 2003) y fueron: pH 3.84, CE 3.22 dS $\mathrm{m}^{-1}, \mathrm{NO}_{3^{-}} 1.50, \mathrm{H}_{2} \mathrm{PO}_{4^{-}} 2.20, \mathrm{Ca}^{2+} 43.30, \mathrm{~K}^{+} 1.10$ y Mg$^{2+} 20.80$ meq L $^{-1}$; y pH 7.82 y CE $0.64 \mathrm{dS} \mathrm{m}^{-1}$, $\mathrm{NO}_{3}-1.60, \mathrm{~K}^{+} 1.18, \mathrm{Ca}^{2+} 4.51, \mathrm{Mg}^{2+} 1.56$ y SO $4^{-}$ 0.05 meq L $^{-1}$; B 0.31, Fe 0.11, Mn 0.034, Cu 0.002 y Zn $0.02 \mathrm{mg} \mathrm{L}^{-1}$; respectivamente. Los resultados del análisis de agua se utilizaron para realizar el ajuste de nutrientes de la solución nutritiva de acuerdo con Steiner (1961).

Los tratamientos fueron cuatro combinaciones de vinazas diluidas en agua de riego, suministrados mediante fertirriego con goteros de $8 \mathrm{~L} \mathrm{~h}^{-1}$, aplicando $3.2 \mathrm{~L} \mathrm{bolsa}^{-1}$ por día en tomate y pepino, y 2.4 $\mathrm{L}$ bolsa $^{-1}$ por día en lechuga, con sus respectivos porcentajes de la solución nutritiva Steiner (Steiner, 1961) y su equivalente de $\mathrm{KNO}_{3^{-}}$(Tabla 2) y como testigo el $100 \%$ de la solución nutritiva $\left(\mathrm{NO}_{3^{-}}{ }^{-12}\right.$, $\mathrm{H}_{2} \mathrm{PO}_{4^{-}} 1, \mathrm{~K}^{+} 7, \mathrm{Ca}^{2+} 9$ y $\mathrm{Mg}^{2+} 4, \mathrm{SO}_{4}^{2-} 7$ meq $\mathrm{L}^{-1}$.) La concentración del $100 \%$ de vinaza en el tratamiento $1 \mathrm{y}$; en los otros tres tratamientos que contenían vinazas, se refiere al volumen máximo de vinaza agregado y que se obtuvo de acuerdo con la concentración de nitrógeno, que fue el elemento menos abundante en la vinaza. Para el tratamiento 1 , el volumen de vinaza fue de $208 \mathrm{~L}$ y se aforó con 792 $\mathrm{L}$ de agua para el volumen final de $1000 \mathrm{~L}$. Debido al bajo contenido de nitrógeno y potasio en la vinaza, en los cuatro tratamientos se agregó el correspondiente de nitrato de potasio y se ajustaron los valores de $\mathrm{pH}$ con ácido nítrico de acuerdo con Urrestarazu (2004) a un rango entre 5.5 y 6.5 .

El volumen de riego aplicado dependió de la etapa fenológica de cada cultivo y de las condiciones climáticas en el interior del invernadero (temperatura diurna promedio $27{ }^{\circ} \mathrm{C}$, nocturna $20{ }^{\circ} \mathrm{C}$ y humedad relativa de $62.8 \%$ ). Las variables climáticas se registraron durante todo el ciclo de cultivo cada 5 min las $24 \mathrm{~h}$ del día, con sensores $\mathrm{HOBO}^{\circledR}$ ProV2 (Onset, EUA). También, se procuró un drenaje de 20 a $30 \%$ respecto al volumen aplicado. Se realizaron las siguientes prácticas de manejo: eliminación de malezas de forma manual una vez por semana, podas de formación en pepino y tomate; además, control de mildiu polvoriento (Pseudoperonospora cubensis) en pepino y tizón tardío (Phytophthora infestans) en tomate con oxicloruro de cobre (Cupravit ${ }^{\circledR}$ ) en dosis de $3 \mathrm{~kg} \mathrm{ha}^{-1}$, el control de mosca blanca (Bemicia tabaci) en tomate se realizó con bifentrina (Binder ${ }^{\circledR}$ ) en dosis de $0.5 \mathrm{Lha}^{-1}$. Las enfermedades y plagas se controlaron de acuerdo con las recomendaciones de Carrasco y Sandoval (2016) para lechuga, Nieto y Velasco (2006) para tomate y López-Elías et al. (2011) en pepino.

Las variables evaluadas se midieron a las 12 semanas después del trasplante (sdt) y fueron; en lechuga, altura de la planta $(\mathrm{cm})$, con una cinta 
Tabla 2. Tratamientos utilizados en la biofertilización de lechuga, tomate y pepino cultivados en invernadero y parámetros fisicoquímicos.

\begin{tabular}{|c|c|c|c|c|c|c|}
\hline \multirow[t]{2}{*}{ Tratamiento } & \multicolumn{2}{|c|}{ Dilución (L) } & \multirow{2}{*}{$\begin{array}{l}\text { SN } \\
(\%)\end{array}$} & \multirow{2}{*}{$\begin{array}{c}\mathrm{KNO}_{3} \\
(\mathrm{~g})\end{array}$} & \multirow[t]{2}{*}{$\mathrm{pH}$} & \multirow{2}{*}{$\begin{array}{c}C E \\
\left(\mathrm{dS} \mathrm{m}^{-1}\right)\end{array}$} \\
\hline & Vinaza & Agua & & & & \\
\hline 1 & $208(100)^{*}$ & 792 & 0 & 693.87 & 6.56 & 0.92 \\
\hline 2 & $166(80)$ & 834 & 20 & 556.71 & 6.96 & 1.19 \\
\hline 3 & $83(40)$ & 917 & 60 & 280.78 & 6.74 & 1.14 \\
\hline 4 & $42(20)$ & 958 & 80 & 140.79 & 6.56 & 1.01 \\
\hline Testigo & - & 1000 & 100 & 303 & 6.81 & 1.85 \\
\hline
\end{tabular}

métrica flexible marca Truper ${ }^{\circledR}$, número de hojas por conteo directo antes del corte, peso fresco y seco (g), mediante una estufa de aire forzado a $70{ }^{\circ} \mathrm{C}$ hasta peso constante y rendimiento total $\left(\mathrm{kg} \mathrm{m}^{-2}\right)$. En tomate, la altura de la planta se midió en $\mathrm{cm}$ con una cinta métrica flexible marca Truper ${ }^{\circledR}$ y se cosecharon siete racimos. El rendimiento total fue la suma de los siete racimos $\left(\mathrm{kg} \mathrm{m}^{-2}\right)$. En pepino, el peso del fruto (g) y rendimiento total $\left(\mathrm{kg} \mathrm{m}^{-2}\right)$ se obtuvo en seis cortes, con una balanza analítica digital (modelo MSL, Marca Metter Toledo $\left.{ }^{\circledR}\right)$.

\section{Análisis de datos}

Se realizó un análisis descriptivo del efecto fitotóxico por medio de los índices de toxicidad de IGN e IER y una prueba de correlación de Pearson para comprobar la relación entre la CE y los valores de IGN e IER. Se comprobaron los supuestos de normalidad (Shapiro-Wilk) y homogeneidad de varianzas (prueba de Levene) de los datos previo al análisis de varianza (ANOVA) para determinar diferencias entre tratamientos en cada cultivo; posteriormente se realizó un análisis de comparación de medias para las variables evaluadas en el cultivo de lechuga, tomate y pepino (Tukey, $\alpha \leq 0.05$ ) (García-Villalpando et al. 2001). En todos los análisis, se usó el paquete estadístico SPSS versión 23 (SPSS 2015).

\section{RESULTADOS}

\section{Experimento 1. Efecto fitotóxico de las vinazas del mezcal en semillas de hortalizas}

En semillas de lechuga, la concentración de
$2.5 \%$ de vinaza del mezcal tuvo baja toxicidad, pero concentraciones superiores a $5 \%$, mostraron toxicidad moderada en el índice de germinación normalizada (Figura 1A) y toxicidad baja para el índice de elongación radical (Figura 1B). A partir del 10\% de concentración de vinaza, el nivel de toxicidad para lechuga fue alto con inhibición completa de la germinación, lo que indica que la vinaza del mezcal en 2.5 y $5 \%$ de concentración disminuye el IGN e IER para las semillas de esta especie, y a partir del $10 \%$ de concentración las inhiben de forma completa.

Para el tomate, las concentraciones menores o igual al $10 \%$ de vinaza, presentaron baja toxicidad, mientras que concentraciones entre 20 y $40 \%$ fueron moderadamente tóxicas y muy tóxicas, respectivamente; mientras que las concentraciones del $80 \mathrm{y}$ $100 \%$ inhibieron la germinaron. Para las semillas de pepino, las bajas concentraciones de vinazas no tuvieron un comportamiento claro, pero la concentración al $40 \%$ de vinaza fue muy tóxica. Tanto en los valores de IGN (Figura 1A) como de IER (Figura 1B) el comportamiento fue similar. En las concentraciones de 80 y $100 \%$ de vinaza hubo germinación de las semillas de pepino, pero el índice IGN y IER fue altamente tóxico y muy tóxico, respectivamente.

\section{Correlación de la salinidad con los índices de toxicidad}

El IGN de las tres especies estudiadas mostró correlación negativa con la $C E(P \leq 0.01)$. Para el IER de lechuga y tomate se observó una correlación negativa con la $C E(P \leq 0.01)$. Para el pepino la correlación entre IER y CE fue baja y no significativa $(P \leq 0.060)$, explicado por la hormesis ante el 

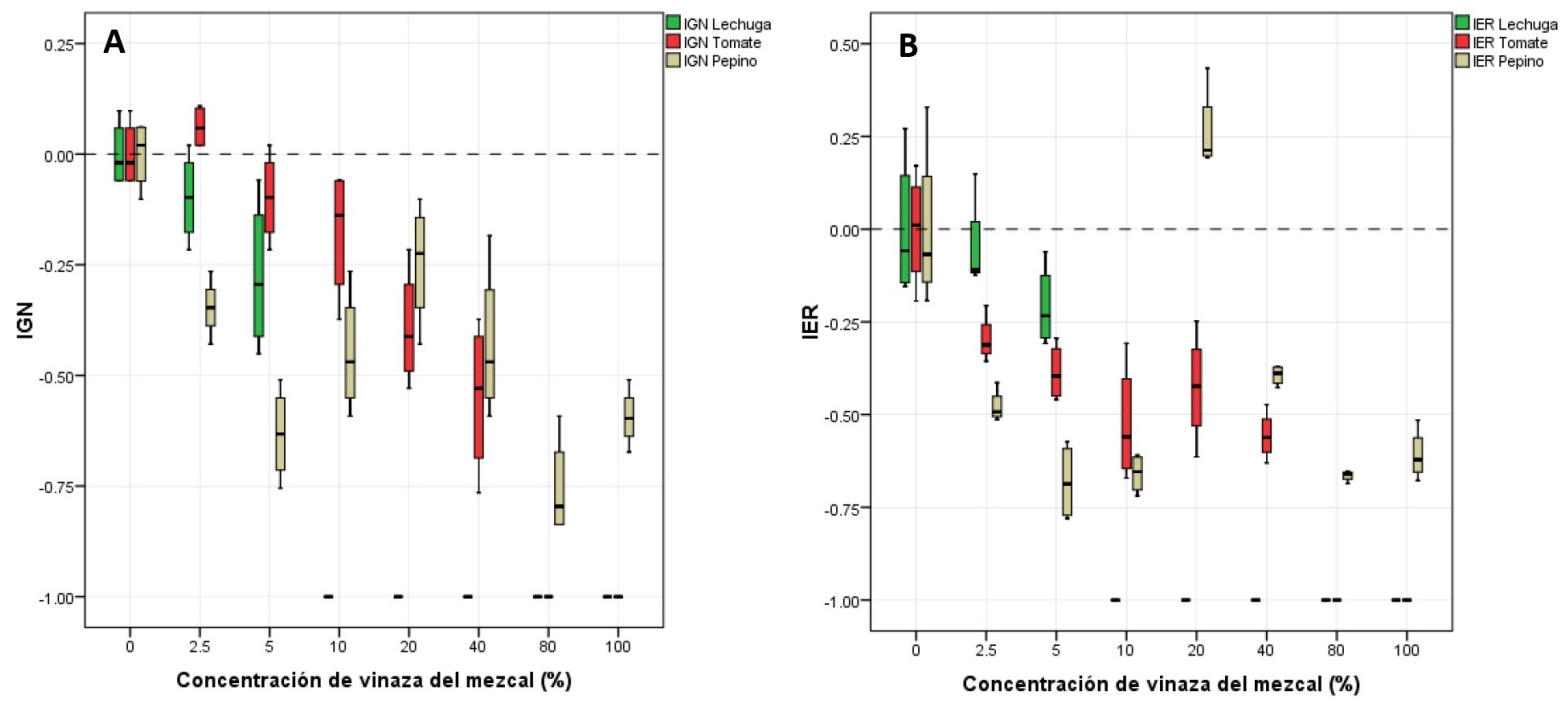

Figura 1. Efecto de las concentraciones de vinazas del mezcal, sobre: A) índice de germinación normalizada (IGN) y B) índice de elongación radical (IER) en semillas de lechuga, tomate y pepino.

$20 \%$ de concentración (Figura 1B). El coeficiente de correlación entre CE y ambos índices fue mayor en el tomate que en lechuga y pepino (Tabla 3). Estos resultados indican que a medida que aumentó la concentración de sales en el medio de germinación, la emergencia y la elongación radical disminuyeron.

\section{Experimento 2. Efecto de las vinazas en el rendimiento en Lechuga}

Se encontraron diferencias significativas $(P \leq$ 0.05 ) entre tratamientos, para todas las variables de lechuga evaluadas. La altura de la planta fue significativamente mayor en T3, que contenía $40 \%$ de vinaza más $60 \%$ de $\mathrm{SN}$. A la vez, este mismo tratamiento, no mostró diferencia significativa con el testigo (100\% SN) para número de hojas, peso fresco y seco por planta y el rendimiento (Tabla 4). Fue en los tratamientos T1 y T4 (100-0 y $20-80 \%$ vinaza y $\mathrm{SN}$, respectivamente, en los que se obtuvieron los valores más bajos en las variables evaluadas.

\section{Tomate}

En el cultivo de tomate, se encontraron diferencias estadísticas significativas $(P \leq 0.05)$ entre tratamientos en la altura de planta y rendimiento. $\mathrm{Pa}$ - ra el testigo y los tratamientos 2 y 3 , con $80-20$ y $40-$ $60 \%$ de vinaza y solución nutritiva, respectivamente, las plantas fueron más altas sin diferencias significativas entre estos tratamientos. Contrariamente, la menor altura se obtuvo con el tratamiento 1, que contenía el $100 \%$ de vinaza, sin solución nutritiva (Tabla 5). El mayor rendimiento del tratamiento 3, sin diferencia estadística con el testigo, indica que se puede usar la vinaza hasta el $40 \%$ sin afectar el rendimiento.

\section{Pepino}

Se encontraron diferencias significativas $(P$ $\leq 0.05)$ entre tratamientos para el peso del fruto y rendimiento de pepino (Tabla 5). Los tratamientos $3 \mathrm{y}$ 4 , con $40-60$ y $20-80 \%$ de vinaza y solución nutritiva, respectivamente; no presentaron diferencias significativas. Los tratamientos 3 y 4 tuvieron los frutos de mayor peso en relación con los demás tratamientos con 318 y $376 \mathrm{~g}$, respectivamente. El mayor rendimiento de frutos lo tuvo el tratamiento testigo que fue diferente estadísticamente a los tratamientos con vinaza, observándose que el rendimiento disminuyó con el incremento de vinaza (Figura 2). 
Tabla 3. Correlaciones entre CE y los índices de toxicidad IGN e IER en las pruebas de germinación de semillas de lechuga, tomate y pepino.

\begin{tabular}{|c|c|c|c|c|c|c|c|}
\hline \multirow{2}{*}{\multicolumn{2}{|c|}{ Correlación }} & \multicolumn{3}{|c|}{ IGN } & \multicolumn{3}{|c|}{ IER } \\
\hline & & Lechuga & Tomate & Pepino & Lechuga & Tomate & Pepino \\
\hline \multirow[t]{2}{*}{$\mathrm{CE}\left(\mathrm{dS} \mathrm{m^{-1 } )}\right.$} & Correlación de Pearson & $-0.665^{\star \star}$ & $-0.955^{\star \star}$ & $-0.576^{\star \star}$ & $-0.663^{\star \star}$ & $-0.893^{\star \star}$ & -0.336 \\
\hline & Sig. (bilateral) & 0.000 & 0.000 & 0.001 & 0.000 & 0.000 & 0.060 \\
\hline
\end{tabular}

Tabla 4. Variables evaluadas de lechuga cultivada con diferentes combinaciones de vinazas y solución nutritiva en invernadero.

\begin{tabular}{|c|c|c|c|c|c|}
\hline \multirow[t]{2}{*}{ Tratamiento } & \multirow{2}{*}{$\begin{array}{l}\text { Altura de planta } \\
\qquad(\mathrm{cm})\end{array}$} & \multirow{2}{*}{$\begin{array}{l}\text { Número de } \\
\text { hojas }\end{array}$} & Peso fresco & Peso seco & \multirow{2}{*}{$\begin{array}{l}\text { Rendimiento } \\
\left(\mathrm{kg} \mathrm{m}^{-2}\right)\end{array}$} \\
\hline & & & \multicolumn{2}{|c|}{ g planta $^{-1}$} & \\
\hline 1 & $29.92 \pm 1.44^{b}$ & $15.12 \pm 0.66^{a b}$ & $392.74 \pm 50.05^{b}$ & $28.14 \pm 4.04^{b}$ & $1.96 \pm 0.73^{b}$ \\
\hline 2 & $31.57 \pm 1.75^{b}$ & $22.32 \pm 0.66^{a}$ & $434.60 \pm 71.70^{b}$ & $30.84 \pm 7.11^{a b}$ & $2.18 \pm 0.49^{b}$ \\
\hline 3 & $39.93 \pm 2.26^{a}$ & $21.60 \pm 1.34^{a}$ & $485.76 \pm 54.79^{a b}$ & $36.22 \pm 5.51^{a b}$ & $2.42 \pm 0.68^{a b}$ \\
\hline 4 & $30.25 \pm 2.83^{b}$ & $13.68 \pm 1.07^{b}$ & $392.10 \pm 45.99^{b}$ & $26.44 \pm 3.97^{b}$ & $1.96 \pm 0.53^{b}$ \\
\hline Testigo & $31.57 \pm 2.73^{b}$ & $15.72 \pm 2.00^{a b}$ & $558.78 \pm 46.78^{a}$ & $40.02 \pm 4.05^{a}$ & $2.80 \pm 0.56^{a}$ \\
\hline DMS & 2.79 & 1.54 & 124.34 & 11.23 & 0.62 \\
\hline CV (\%) & 12.96 & 21.52 & 22.17 & 32.71 & 22.17 \\
\hline
\end{tabular}

$\mathrm{n}=5.0 . \pm$ : desviación estándar. Letras distintas en una misma columna indican diferencias significativas $(\mathrm{P} \leq$ 0.05). DMS = diferencia mínima significativa. $C V$ = coeficiente de variación.

Tabla 5. Variables de crecimiento y rendimiento de tomate y pepino con diferentes combinaciones de vinazas y solución nutritiva.

\begin{tabular}{ccccc}
\hline \multirow{2}{*}{ Tratamientos } & \multicolumn{2}{c}{ Tomate } & \multicolumn{2}{c}{ Pepino } \\
\cline { 2 - 5 } & Altura de planta $(\mathrm{cm})$ & Rendimiento $\left(\mathrm{kg} \mathrm{m}^{-2}\right)$ & Peso de fruto & -1 \\
& $95.80 \pm 10.12^{b}$ & $7.74 \pm 0.62^{b}$ & $254.71 \pm 27.22^{b}$ & Rendimiento $\left(\mathrm{kg} \mathrm{m}^{-2}\right)$ \\
\hline 1 & $110.40 \pm 10.48^{a b}$ & $7.93 \pm 0.48^{b}$ & $267.09 \pm 31.92^{b}$ & $9.10 \pm 2.31^{c}$ \\
2 & $104.00 \pm 11.04^{a b}$ & $9.14 \pm 0.55^{a}$ & $318.41 \pm 46.87^{a b}$ & $14.84 \pm 1.99^{b}$ \\
3 & $100.00 \pm 12.02^{b}$ & $7.56 \pm 0.76^{b}$ & $376.82 \pm 72.80^{a}$ & $15.70 \pm 2.1^{b}$ \\
4 & $121.20 \pm 18.94^{a}$ & $8.58 \pm 0.59^{a}$ & $283.98 \pm 27.88^{b}$ & $19.72 \pm 1.45^{a}$ \\
Testigo & 19.10 & 2.48 & 66.08 & 7.23 \\
DMS & 14.24 & 19.89 & 20.20 & 35.32 \\
CV \% & $\mathrm{n}=5.0 . \pm:$ desviación estándar. Letras distintas en una misma columna indican diferencias significativas $(\mathrm{P} \leq 0.05)$. \\
DMS = diferencia mínima significativa. CV = coeficiente de variación.
\end{tabular}

\section{DISCUSIÓN}

\section{Experimento 1. Efecto fitotóxico de las vinazas del mezcal en semillas de hortalizas}

Las semillas evaluadas tuvieron comportamientos diferentes en el índice de toxicidad, las de lechuga fueron las más afectadas, seguidas del tomate y el pepino. Para la lechuga, la afectación se tuvo con $2.5 \%$ de vinaza, mientras que para el tomate y pepino fue al 5\%. La inhibición de la germinación de las semillas por la vinazas, se debe al elevado contenido de sales, que impiden la imbibición de las semillas por la presión osmótica que ejercen en el medio (Meza et al. 2007), por lo que el embrión puede tardar más tiempo en activar enzimas como la arginina, encargada de romper la latencia (Goykovic y Saavedra 2007). Sin embargo, en pepino la activación de arginina se da incluso ante estrés por salinidad, el cual es clave para formar enzimas que hidrolizan carbohidratos, lípidos y proteínas presentes en los cotiledones, que nutren a la plántula en su etapa pre-autótrofa (Du et al. 2021). Por otro lado, la tolerancia a la salinidad es menor en semillas de lechuga (Gerber et al. 2017), lo cual explica las diferencias en los valores de los índices de germinación y elongación radical obtenidos en este estudio.

Para el pepino, el IER indica estimulación del crecimiento radicular $u$ hormesis al $20 \%$ de concentración de vinazas (Figura 1B). Al respecto, el contenido de fenoles y ácidos orgánicos de las vinazas del mezcal (Robles-González et al. 2018) pueden actuar como estimuladores de crecimiento en dosis 


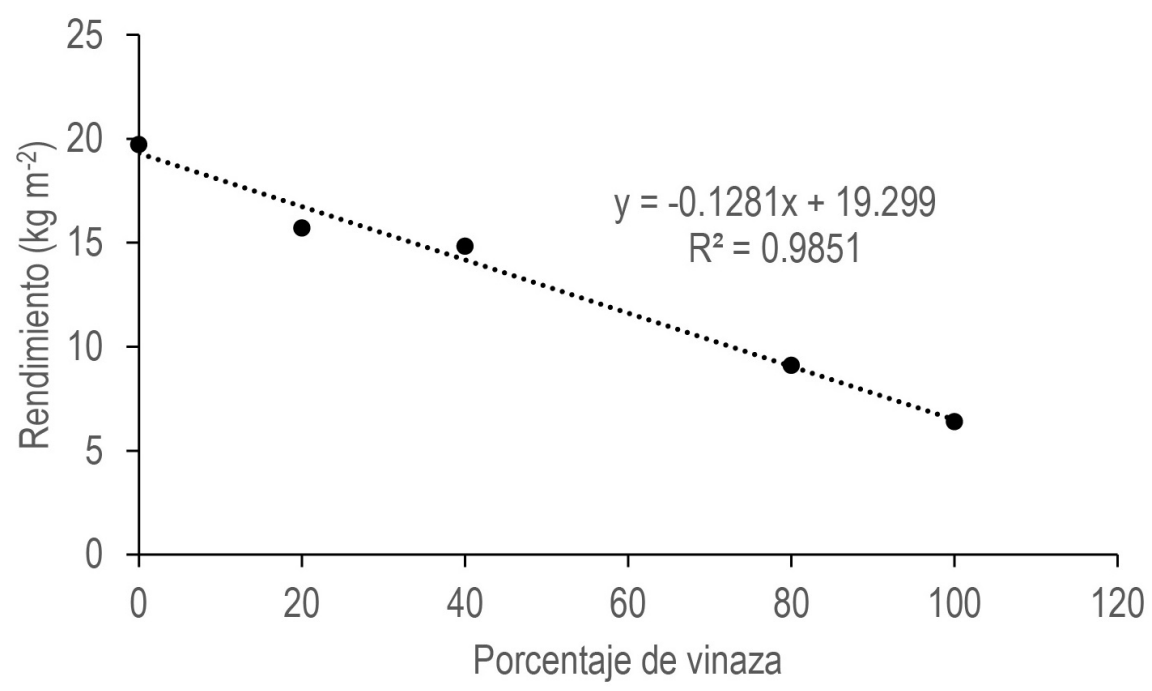

Figura 2. Rendimiento de pepino como respuesta a las combinaciones de vinazas y solución nutritiva en invernadero.

bajas (Jasso-de Rodríguez et al. 2019) y tóxicas en dosis altas (López-Diazguerrero et al. 2013, Sousa et al. 2019), sobre todo en los puntos de crecimiento radical (Viveros-Legorreta et al. 2018). Por el contrario, la concentración de sales puede tener un efecto inhibitorio en la germinación de semillas de algunas especies (Da-Ros et al. 2018) y estimulante en otras, dependiendo de su tolerancia a la salinidad (Urrestarazu 2004). El índice más sensible fue el IER con menor variabilidad en los resultados, como se ha demostrado en otros estudios (Bagur-González et al. 2011). Las vinazas en concentraciones superiores al $2.5 \%$ son tóxicas para las tres especies, siendo el pepino la especie más tolerante y la lechuga la más sensible.

\section{Experimento 2. Efecto de las vinazas en el rendimiento en Lechuga}

La altura de la planta de lechuga alcanzó 39.93 $\mathrm{cm}$ a las $12 \mathrm{sdt}$, superior a los $32.00 \mathrm{~cm}$ reportado por Martínez et al. (2015) en lechugas cv. Roma cultivadas sobre el suelo con fertilización convencional bajo invernadero a las $11 \mathrm{sdt}$, y también superior a los resultados obtenidos por Dos Santos et al. (2013), al evaluar el efecto de las vinazas de caña de azúcar (Saccharum oficcinarum L.) con fertilización hidropó- nica de lechuga, con altura de $13.90 \mathrm{~cm}$ a las seis sdt al aplicar una dilución del $10 \%$ de vinazas. Esto se puede deber a que en el presente experimento se tuvo el doble de semanas que el reportado por Dos Santos et al. (2013). Las 21.60 hojas obtenidas en el tratamiento 3, fue menor a las 45 hojas alcanzado por Martínez et al. (2015) con fertilización convencional bajo invernadero a las $11 \mathrm{sdt}$. El mayor peso fresco (558.78 y $485.76 \mathrm{~g} \mathrm{planta}^{-1}$ ), obtenidos con el tratamiento testigo y con el T3, respectivamente, fue menor a lo reportado por Martínez et al. (2015) para lechuga (810.91 $\mathrm{g} \mathrm{planta}^{-1}$ ) a las $11 \mathrm{sdt}$; mientras que el rendimiento de 2.80 y $2.42 \mathrm{~kg} \mathrm{~m}^{-2}$ con estos mismos tratamientos, fueron menores al obtenido por Martínez et al. (2015) con $7.80 \mathrm{~kg} \mathrm{~m}^{-2}$ con la misma densidad de siembra. Estas diferencias en el rendimiento se deben, evidentemente, al mayor peso fresco por planta que dichos autores obtuvieron en cultivo sobre suelo con fertilización convencional, en invernadero con control pasivo del clima y en un ambiente favorable para el cultivo de lechuga. Estos resultados evidencian la viabilidad de utilizar las vinazas de la agroindustria del mezcal, en diluciones del $40 \%$ para el cultivo de lechuga, combinada con el $60 \%$ de la solución nutritiva. 


\section{Tomate}

Los tratamientos 2 y 3 , que contenían 80-20 y $40-60 \%$ de vinaza y solución nutritiva, respectivamente, estimularon que las plantas de tomate alcanzaran alturas de 110.40 y $104.00 \mathrm{~cm}$, valores que son estadísticamente similares al testigo que tuvo $121.20 \mathrm{~cm}$. Siendo estos resultados similares a lo reportado por Jasso-de Rodríguez et al. (2019) para tomate tipo saladette en el mismo periodo de tiempo después del trasplante $(120 \mathrm{~cm})$ y mayor a lo reportado por Roque-Enriquez et al. (2021) quienes tuvieron $86.80 \mathrm{~cm}$ de altura en plantas de tomate cultivadas en bolsas de polietileno de $10 \mathrm{~L}$ con una mezcla de peat moss y perlita, irrigadas con solución nutritiva Steiner. Para el rendimiento se cosecharon siete racimos, los mayores resultados se tuvieron con los tratamientos T3 y el testigo, los cuales produjeron 9.14 y $8.58 \mathrm{~kg} \mathrm{~m}^{-2}$ equivalente a 2.40 y 2.15 $\mathrm{kg}_{\text {planta }}{ }^{-1}$, respectivamente; resultados que fueron similares a los $9.30 \mathrm{~kg} \mathrm{~m}^{-2}$ obtenidos por Jasso-de Rodríguez et al. (2019) en cultivo hidropónico de tomate sobre bolsas de plástico con perlita y peat moss $1: 1$, pero inferiores a los 11.17 y $13.60 \mathrm{~kg} \mathrm{~m}^{-2}$ obtenidos por García-León et al. (2018) en tomates de variedades tradicionales de crecimiento indeterminado cultivados sobre suelo y fertilizados de manera convencional. Estas diferencias se deben al tipo de variedades y el sustrato utilizado ya que en el presente estudio se usó una variedad de crecimiento determinado y sustrato a base de bagazo de agave compostado en bolsas de polietileno. Los resultados obtenidos, muestran la posibilidad de utilizar la combinación del $40 \%$ de vinazas con $60 \%$ de solución nutritiva para el cultivo de tomate sin afectar el rendimiento.

\section{Pepino}

El mayor peso de frutos se obtuvo con los tratamientos 3 y 4 con 318.71 y $376.82 \mathrm{~g}$, respectivamente; los cuales son similares a lo reportado por López-Elías et al. (2011) con $337.00 \mathrm{~g}$ en pepino del mismo tipo que el presente estudio cultivado en suelo bajo invernadero y fertilizado de manera convencional, pero superiores a los $205.50 \mathrm{~g}$ alcanzado por Del Busto et al. (2018). El rendimiento de esta hortaliza fue significativamente superior para el testigo, que produjo $19.72 \mathrm{~kg} \mathrm{~m}^{-2}$. Para los tratamientos que contenían vinazas en su solución nutritiva, se tuvieron dos grupos significativamente diferentes entre T3 y T4, y entre T1 y T2, con rendimientos intermedios $\left(14.84\right.$ y $15.70 \mathrm{~kg} \mathrm{~m}^{-2}$ ) y bajos $(6.39$ y $9.10 \mathrm{~kg}$ $\mathrm{m}^{-2}$ ), respectivamente. Los rendimientos de pepino de los tratamientos T3 y T4, estuvieron cercanos a los $16.30 \mathrm{~kg} \mathrm{~m}^{-2}$ encontrados por López-Elías et al. (2011) en siembra directa bajo invernadero. Si bien, el tratamiento T2 que contenía $80 \%$ de aporte de nutrientes de vinazas del mezcal obtuvo rendimiento bajo en el presente estudio, se acerca a lo reportado por Del Busto et al. (2018) con $11.38 \mathrm{~kg} \mathrm{~m}^{-2}$ de pepino americano en siembra directa y fertilizado de manera convencional. De manera general, el rendimiento de esta especie vegetal, disminuyó de forma lineal $\left(R^{2}=0.98, P \leq 0.01\right)$ y proporcional al aumento del porcentaje de vinaza en la solución nutritiva (figura 2).

Los nutrientes disueltos en las vinazas, liberados de las estructuras orgánicas del agave durante la cocción y fermentación, como nitrógeno (Robles-González et al. 2018), potasio (Silva et al. 2019), calcio, sodio, magnesio, cobre, hierro, zinc (Sánchez-Lizárraga et al. 2018) se presentan en forma asimilable por las plantas y contribuyeron a complementar las soluciones nutritivas utilizadas en el cultivo de lechuga, tomate y pepino de este estudio. Pero al aumentar la concentración de vinaza, como en los tratamientos T1 y T2, los compuestos fenólicos, alcoholes y ácidos orgánicos presentes (Sánchez-Lizárraga et al. 2018) pueden afectar los puntos de elongación radical, como lo obtenido en el experimento $1 \mathrm{y}$ reportado por Sousa et al. (2019), así como afectar el desarrollo del cultivo y su rendimiento. Para reducir los efectos adversos de la vinaza del mezcal en hortalizas, sería recomendable realizar un pretratamiento para eliminar o disminuir la concentración de dichos compuestos nocivos; ya sea por medios físicos como la ósmosis inversa (Silva et al. 2019) o por medios biológicos como la digestión anaeróbica (Morán-Salazar et al. 2016). 


\section{CONCLUSIONES}

La concentración del $2.5 \%$ de vinaza del mezcal fue tóxica para las semillas de lechuga y mayor del $10 \%$ inhibió su germinación. Para las semillas de tomate y pepino el $20 \%$ fue tóxico, mientras que el índice de germinación y de elongación radical, disminuyeron al aumentar la concentración de vinazas. En semillas de tomate y pepino el 2.5 y $20 \%$, respectivamente provocaron hormesis; mientras que concentraciones mayores del 10\% inhibieron la germinación de las semillas de lechuga. El mayor rendimiento de lechuga y tomate se tuvo con $40+60 \%$ de vinazas y solución nutritiva, mientras que, en pepino, se encontraron los mayores rendimientos con $40+60$ y $20+$ $80 \%$ de vinazas y solución nutritiva, pero fue menor que el que tuvo el testigo. El rendimiento de pepino disminuyó de forma lineal y proporcional al aumentar el porcentaje de vinazas del mezcal como complemento de la solución nutritiva.

\section{AGRADECIMIENTOS}

Al Instituto Politécnico Nacional, por el financiamiento otorgado al proyecto SIP- 20200681, al CONACYT por la beca 565495 para estudios Doctorales, a la Ing. Gabriela Robles por apoyar en establecimiento del experimento y al señor Valeriano Ortega por proveer las vinazas y el bagazo de agave utilizado en este estudio.

\section{LITERATURA CITADA}

AOAC (2003) Official Methods of Analysis. 17th edn. Vol.I.17th ed. Association of Analytical Washington, DC, USA.

Bagur-González MG, Estepa-Molina C, Martin-Peinado F, Morales-Ruano S (2011) Toxicity assessment using Lactuca sativa L. bioassay of the metal(loid)s $\mathrm{As}, \mathrm{Cu}, \mathrm{Mn}, \mathrm{Pb}$ and $\mathrm{Zn}$ in soluble-in-water saturated soil extracts from an abandoned mining site. Journal of Soils and Sediments 11: 281-289.

Beltrán FJ, Alvarez PM, Rodriguez EM, Garcia-Araya JF, Rivas J (2001) Treatment of high strength distillery wastewater (Cherry stillage) by integrated aerobic biological oxidation and ozonation. Biotechnology Progress 17: 462-467.

Carrasco G, Sandoval C (2016) Manual práctico del cultivo de la lechuga. Mundi-prensa. México. 148p.

Castro-Lizazo I, Diez-Rojo MÁ, González-López MR, López-Pérez JA (2021) Germinación, fitotoxicidad y efecto nematicida de la vinaza de vino, un subproducto de la industria vinícola. Revista de Protección Vegetal 36: $1-9$.

Castro-Rivera R, Solís-Oba MM, Chicatto-Gasperín V, Solís-Oba A (2020) Biogas production through co-digestion of cattle waste and agricultural residues of tomato cultivation (Solanum lycopersicum L.). Revista Internacional de Contaminacion Ambiental 36: 529-539.

CRM (2019) El Mezcal. La cultura líquida de México. Número 2. Febrero 2019. Oaxaca de Juárez. http://www. crm.org.mx/periodico/PDF/Revista_El_Mezcal2.pdf. Fecha de consulta: 25 de junio de 2019.

Da Ros C, Libralato G, Ghirardini AV, Radaelli M, Cavinato C (2018) Assessing the potential phytotoxicity of digestate from winery wastes. Ecotoxicology and Environmental Safety 150: 26-33.

Del Busto AC, Santana BY, Gonzalez BF, Dominguez GJ, López QY, Diaz BM, Hidalgo VY, Cabrera RJE (2018) Agronomic evaluation of cucumber hybrids under house of culture, Pinar del Río, Cuba. Centro Agrícola 45: 88-91.

Dos Santos JD, Lopes da Silva AL, da Luz Costa J, Scheidt GN, Novak AC, Sydney EB, Soccol CR (2013) Development of a vinasse nutritive solution for hydroponics. Journal of Environmental Management 114: 8-12. 
Du C, Li H, Liu C, Fan H (2021) Understanding of the postgerminative development response to salinity and drought stresses in cucumber seeds by integrated proteomics and transcriptomics analysis. Journal of Proteomics 232: 104062. DOI: 10.1016/J.JPROT.2020.104062.

García-León Á, Robledo-Torres V, Mendoza-Villarreal R, Ramírez-Godina F, Valdez-Aguilar LA, Gordillo-Melgoza FA (2018) Producción de variedades tradicionales de tomate con acolchado en invernadero. Ecosistemas y Recursos Agropecuarios 5: 303-308.

García-Villalpando JA, Castillo-Morales A, Ramírez-Guzmán ME, Rendón-Sánchez G, Larqué-Saavedra MU (2001) Comparación de los procedimientos de Tukey, Duncan, Dunnett, Hsu y Bechhofer para selección de medias. Agrociencia 35: 79-86.

Gerber MD, Lucia T, Correa L, Neto JEP, Correa ÉK (2017) Phytotoxicity of effluents from swine slaughterhouses using lettuce and cucumber seeds as bioindicators. Science of The Total Environment 592: 86-90.

Goykovic V, Saavedra G (2007) Algunos efectos de la salinidad en el cultivo del tomate y prácticas agronómicas de su manejo. Idesia 25: 47-58.

SPSS (2015) IBM SPSS Statistics for Windows, Version 23.0. IBM Corp. Released. Armonk, NY: IBM Corp.

Jasso-de Rodríguez D, Alfonso-Cuevas CF, Rodriguez-García R, Ramírez H, Díaz-Jiménez L, Villarreal-QuintaniIla JA, Juárez-Maldonado A (2019) Extractos de plantas del semidesierto en la inducción del crecimiento de tomate (Lycopersicon esculentum Mill). Ecosistemas y Recursos Agropecuarios 7: e2342. DOI: 10.19136/ era.a7nl.2342.

López-Diazguerrero, NE, González PVY, Hernández-Bautista RJ, Alarcón-Aguilar A, Luna-López A, Mina KF (2013) Hormesis: lo que no mata, fortalece. Gaceta Médica de México 149: 438-447.

López-Elías J, Rodríguez JC, Huez LMA, Garza OS, Jiménez LJ, Leyva EEI (2011) Production and quality of cucumber (Cucumis sativus L.) under greenhouse conditions using two pruning systems. Idesia 29: 21-27.

López-López A, Davila-Vazquez g, León-Becerril E, Villegas-García E, Gallardo-Valdez J (2010) Tequila vinasses: Generation and full scale treatment processes. Reviews in Environmental Science and Bio/Technology 9: 109-116.

López-Velarde SM, Ventura-Ramos E, Rodríguez-Morales JA, Oliver H (2020) Effect of inoculum source on the anaerobic digestion of mezcal vinasses at different substrate-inoculum ratios. Revista Internacional de Contaminacion Ambiental 36: 81-95.

Martínez-Damián MT, Cano-Hernández R, Moreno-Pérez E, Del Castillo FS, Cruz-Álvarez O (2019) Effect of preharvest growth bioregulators on physicochemical quality of saladette tomato. Revista Chapingo Serie Horticultura 25: 29-43.

Martínez-Gutiérrez GA, Íñiguez-Covarrubias G, Ortiz-Hernández YD, López-Cruz JY, Bautista-Cruz MA (2013) Tiempos de apilado del bagazo del maguey mezcalero y su efecto en las propiedades del compost para sustrato de tomate. Revista Internacional de Contaminación Ambiental 29: 209-216.

Martínez CG, Lara HA, Padilla BLE, Luna FM, Avelar MJJ, Lamas LJJ (2015) Evaluación técnica y financiera del cultivo de lechuga en invernadero, como alternativa para invierno. Terra Latinoamericana 33: 251-260.

Mendes PM, Ribeiro JA, Martins GA, Lucia T, Araujo TR, Fuentes-Guevara MD, Corrêa ÉK (2021) Phytotoxicity test in check: Proposition of methodology for comparison of different method adaptations usually used worldwide. Journal of Environmental Management 291. DOI: 10.1016/J.JENVMAN.2021.112698.

Meza N, Arizaleta M, Bautista D (2007) Efecto de la salinidad en la germinación y emergencia de semillas de parchita (Passiflora edulis f. flavicarpa). Revista de la Facultad de Agronomía 24: 69-80. 
Morán-Salazar RG, Sánchez-Lizárraga AL, Rodriguez-Campos J, Davila-Vazquez G, Marino-Marmolejo E N, Dendooven L, Contreras-Ramos SM (2016) Utilization of vinasses as soil amendment: consequences and perspectives. SpringerPlus 6: 1007. DOI: 10.1186/s40064-016-2410-3.

Nieto A, Velasco H (2006) Cultivo de Jitomate en Hidroponía e Invernadero. Segunda ed. México. Departamento de Fitotecnia, Universidad Autónoma de Chapingo. 130p.

Robles-González V, Galindez-Mayer J, Rinderknech-Seijas N, Poggi-Varaldo HM (2012) Treatment of mezcal vinasses: A review. Journal of Biotechnology 157: 524-546.

Robles-González V, Poggi-Varaldo HM, Galíndez-Mayer J, Ruiz-Ordaz N (2018) Combined treatment of mezcal vinasses by ozonation and activated sludge. Water Environment Research 90: 1985-1996.

Rodríguez-Félix E, Contreras-Ramos SM, Davila-Vazquez G, Rodríguez-Campos J, Marino-Marmolejo EN (2018) Identification and quantification of volatile compounds found in vinasses from two different processes of Tequila production. Energies 11: 490. DOI: 10.3390/en11030490.

Rodríguez A, De La Cerna C (2017) El mezcal, su producción y tratamiento de residuos. Alianzas y Tendencias 2: $10-14$.

Rodríguez-Romero JA, Robles-Salazar AC, Ruíz-Picos RA, López-López E, Sedeño-Díaz EJ, Dorantes-Rodríguez A (2014) Índices de germinación y elongación radical de lactuca sativa en el biomonitoreo de la calidad del agua del rio chalma. Revista Internacional de Contaminación Ambiental 30: 307-316.

Roque-Enriquez A, Delgado-Ortiz J, Beltrán-Beache M, Ochoa-Fuentes Y, Cerna-Chávez E (2021) Parámetros agronómicos del tomate (Solanum lycopersicum L.) inoculado con "Candidatus Liberibacter solanacearum" y tratados con fosfitos. Ecosistemas y Recursos Agropecuarios 8: e2552. DOI: 10.19136/ERA.A8N1.2552.

Sánchez-Lizárraga AL, Arenas-Montaño V, Marino-Marmolejo EN, Dendooven L, Velazquez-Fernandez JB, Davila-Vazquez G, Contreras-Ramos SM (2018) Vinasse irrigation: effects on soil fertility and arbuscular mycorrhizal fungi population. Journal of Soils and Sediments 18: 3256-3270.

Silva GA, Ferreira SL, de Souza GR, da Silva JA, Pagliuso JD (2019) Utilization of a new approach for the potassium concentration of sugarcane vinasse by reverse osmosis: case study. International Journal of Environmental Science and Technology 16: 6441-6446.

Sousa RM, Amaral C, Fernandes JMC, Fraga I, Semitela S, Braga F, Sampaio A (2019) Hazardous impact of vinasse from distilled winemaking by-products in terrestrial plants and aquatic organisms. Ecotoxicology and Environmental Safety 183: 109493. DOI: 10.1016/J.ECOENV.2019.109493.

Steiner AA (1961) A universal method for preparing nutrient solutions of a certain desired composition. Plant and Soil 15: 134-154.

Urrestarazu M (2004) Tratado de cultivo sin suelo. 3ra edición. Mundiprensa. Almería, España. 914p.

Visconti F, De Paz JM (2018) Cómo conocer la salinidad del suelo mediante medidas de conductividad eléctrica. Levante Agrícola 441: 98-103.

Viveros-Legorreta JL, Sarma S, Guerrero-Zúñiga LA, Rodríguez-Dorantes A (2018) Bioassay of the effect of phenols produced by myriophyllum aquaticum culture on lactuca sativa. Hidrobiologica 28: 109-119.

Wang W (1991) Literature review on higher plants for toxicity testing. Water, Air, and Soil Pollution 59: 381-400. 